\title{
X-ray measured metallicities of the intra-cluster medium: a good measure for the metal mass?
}

\author{
W. Kapferer, T. Kronberger, J. Weratschnig, and S. Schindler
}

\author{
Institut für Astro- und Teilchenphysik, Universität Innsbruck, Technikerstrasse 25, 6020 Innsbruck, Austria \\ e-mail: wolfgang.e.kapferer@uibk.ac .at
}

Received 28 March 2007 / Accepted 10 July 2007

\begin{abstract}
Aims. We investigate whether X-ray observations map heavy elements in the Intra-Cluster Medium (ICM) well and whether the X-ray observations yield good estimates for the metal mass, with respect to predictions on transport mechanisms of heavy elements from galaxies into the ICM. We further test the accuracy of simulated metallicity maps.

Methods. We extract synthetic X-ray spectra from $N$-body/hydrodynamic simulations including metal enrichment processes, which we then analyse with the same methods as are applied to observations. By changing the metal distribution in the simulated galaxy clusters, we investigate the dependence of the overall metallicity as a function of the metal distribution. In addition we investigate the difference of X-ray weighted metal maps produced by simulations and metal maps extracted from artificial X-ray spectra, which we calculate with SPEX2.0 and analyse with XSPEC12.0.

Results. The overall metallicity depends strongly on the distribution of metals within the galaxy cluster. The more inhomogeneously the metals are distributed within the cluster, the less accurate is the metallicity as a measure for the true metal mass. The true metal mass is generally underestimated by X-ray observations. The difference between the X-ray weighted metal maps and the metal maps from synthetic X-ray spectra is on average less than $7 \%$ in the temperature regime above $T>3 \times 10^{7} \mathrm{~K}$, i.e. X-ray weighted metal maps can be well used for comparison with observed metal maps. Extracting the metal mass in the central parts $(r<500 \mathrm{kpc})$ of galaxy clusters with X-ray observations results in metal mass underestimates up to a factor of three.
\end{abstract}

Key words. hydrodynamics - methods: numerical - galaxies: intergalactic medium

\section{Introduction}

Since the first observations of the $7 \mathrm{keV}$ iron line feature in the 1970's by Mitchell et al. (1976) it has been evident that the intra-cluster medium (ICM) does contain gas already processed by stars. X-ray spectra are the only measure for the metallicity of the ICM. With X-ray observatories like XMM-Newton or Chandra it is nowadays possible to extract metallicities in certain regions of a galaxy cluster and construct metallicity profiles and X-ray weighted metallicity maps (Pratt et al. 2006; Durrett et al. 2005). In addition to observations galaxy cluster simulations including gas and dark matter (DM) physics are an ideal tool to investigate the evolution of these systems. By including different enrichment processes it is possible to trace the metals in the ICM. Recent work in this field (Cora 2006; Kapferer et al. 2006; Kapferer et al. 2007; Domainko et al. 2006; Moll et al. 2007; Springel \& Hernquist 2003) are able to reproduce the inhomogeneous distribution of metals within the ICM as observed (Durret et al. 2005). Given the large mass fraction of the ICM in a cluster (15-20\%) compared to the mass fraction of the galaxies (3-5\%) a lot of metals must have been produced within the galaxies and then been transported together with part of the interstellar medium (ISM) into the ICM. X-ray observations can also distinguish the lines of different elements, e.g. elements like $\mathrm{Si}, \mathrm{S}, \mathrm{O}$ from core collapse supernovae or the elements $\mathrm{Fe}$ and $\mathrm{Ni}$ from supernovae Ia (Baumgartner et al. 2005; Ettori et al. 2002; Sanders et al. 2004; Finoguenov et al. 2002) and can hence give information on the origin of the metals and interaction in clusters. From these observations we know (e.g. Mushotzky 1999), that most of the metals are in the ICM and not in stars. Thus, to trace metal formation and evolution it is crucial to get correct estimates for the metal content in the ICM.

Three dimensional hydrodynamic galaxy cluster simulations give us an estimate of the "true" metallicity distribution within simulated galaxy clusters. By constructing artificial X-ray profiles and maps it is possible to test, whether metallicities obtained by X-ray spectra are a good measure for the real metal content in the ICM or not.

The paper is structured as follows. First we compare X-ray weighted metal maps with metal maps obtained from synthetic spectra, which we construct with SPEX 2.0 (Kaastra et al. 1996) and investigate with XSPEC 12.0 (Dorman et al. 2003). Then we compare different heavy element distributions in the ICM and their spectroscopic appearance. We give constraints on the accuracy of spectroscopic metallicities as a measure of the real metal content in the ICM. Throughout the paper we assume solar ratios (Anders \& Grevesse 1989).

\section{The model clusters}

We take two realistic ICM distributions from model clusters for all further calculations into account. We apply a standard $\Lambda$ CDM simulation with gas physics and constraints random fields (see Kapferer et al. 2007; Kapferer et al. 2006; Domainko et al. 2006; and Schindler et al. 2005, for more details) to obtain a reasonable ICM gas distribution. The first model cluster is a merging galaxy 
cluster, hereafter model cluster A, whereas the second system is a more massive galaxy cluster, hereafter model cluster B. The properties of the model galaxy clusters are as follows:

- Model cluster A: The cluster forms at $z \sim 1.5$ and has two major merger events at $z=0.8$ and $z=0.5$. The final total mass is $1.5 \times 10^{14} M_{\odot}$ within a sphere of radius $1 \mathrm{Mpc}$.

- Model cluster B: The formation redshift for this cluster is $z \sim 1.7$. It shows four minor merger events a $z=1.4, z=$ $1.1, z=0.5$ and $z=0.3$. The cluster has a final mass of $3.4 \times 10^{14} M_{\odot}$ within a sphere of radius $1 \mathrm{Mpc}$.

The galaxy cluster simulations have two enrichment processes included, namely galactic winds and ram-pressure stripping. The enrichment process description reproduces the metallicity profile of non-cooling flow and massive galaxy clusters (see Kapferer et al. 2007, for more details about the enrichment history and a detailed analysis on the metal distribution). With these two realistic models for ICM distribution we performed all analysis on the difference of the true metal mass, which we know exactly from the simulation, and the metal mass we would obtain from X-ray observations of our model clusters.

\section{How to construct $X$-ray weighted metallicity maps}

So far X-ray weighted metal maps where used to obtain metal maps from simulations. We want to test here how accurate these maps are compared to maps, that are obtained by using spectra. The total emissivity by thermal Bremsstrahlung for the ICM above $T>3 \times 10^{7} \mathrm{~K}$ is approximately given by

$$
\epsilon^{f f} \sim 1.4 \times 10^{-27} T^{1 / 2} n_{\mathrm{e}} n_{\mathrm{i}} Z^{2} g_{\mathrm{B}} \quad\left[\mathrm{erg} \mathrm{cm}^{-3} \mathrm{~s}^{-1}\right],
$$

where $T$ is the ICM gas temperature, $n_{\mathrm{e}}, n_{\mathrm{i}}$ are the number densities of electrons and ions, $Z$ is the number of protons of the bending charge and $g_{\mathrm{B}}$ the frequency averaged Gaunt factor. The emissivity is mainly dominated by the density of the ICM. Besides the continuum emission several emission lines in the ICM can be observed. The most prominent lines in the ICM are typically in the $7 \mathrm{keV}$ iron line complex. These lines origin from many stages of ionisation of iron.

The power emitted by the most prominent emission lines in the ICM, the iron $\mathrm{K}$ line complex, which is a resonance line, is given by

$$
\begin{aligned}
P_{j k}= & 8.63 \times 10^{6}\left(n_{e} n_{h} A_{e l} X_{i}(t) E_{j k}\right) \frac{\exp \left(-E_{g j} / k T\right)}{T^{1 / 2}} \\
& \times \frac{8 \pi}{\sqrt{(} 3)} f_{g j}^{\prime} \bar{g}(T) \quad\left[\operatorname{erg~cm}^{-3} \mathrm{~s}^{-1}\right]
\end{aligned}
$$

where $A_{e l}$ is the abundance of the element $e l$ relative to hydrogen and $X_{i}$ the fraction of the ion $i, E_{j k}$ the transition energy from level $j$ to $k, E_{g j}$ the transition energy from ground level $g$ to $j$, the averaged Gaunt factor $\bar{g}(T)$ and $f_{g j}^{\prime}$ the effective oscillator strength. For more details see Sutherland \& Dopita (1993) and references therein. Again the dependence of the emission on the density of the ICM and the temperature of the gas is evident. For a given set of fixed ICM density and iron abundance the temperature dependence of the emission ratio of a given iron line and the bremsstrahlung continuum can be written as

$R(T)=\frac{P_{i k}}{\epsilon^{f f}} \approx \Omega \frac{\exp (A / T)}{T}$

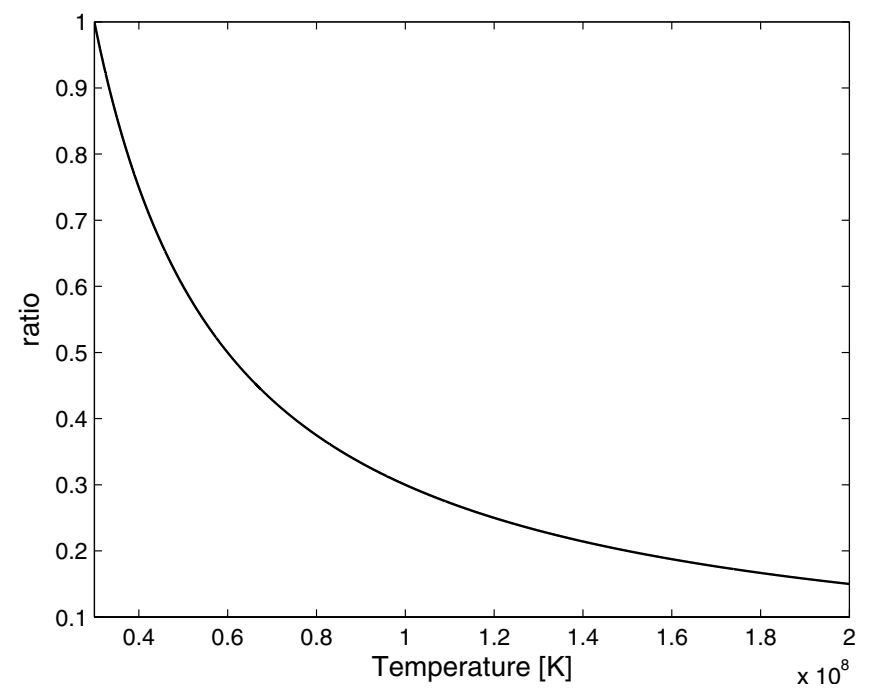

Fig. 1. The temperature dependency of Eq. (3), i.e. the ratio of the emissivity for a given line and the thermal bremsstrahlung continuum in the temperature range $0.3-2 \times 10^{8} \mathrm{~K}$.
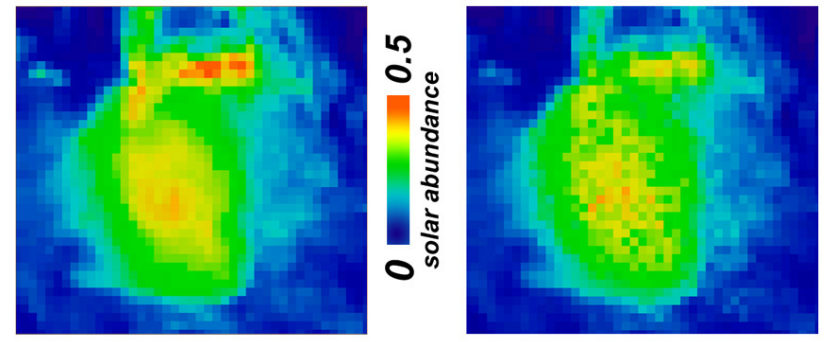

Fig. 2. Left: X-ray weighted metal map of the model galaxy cluster at $z=0$, the metallicity in each cell is weighted by the X-ray emission. Right: synthetic metal map, using a spectrum for each cell. The image has $1.5 \mathrm{Mpc} h^{-1}$ on the side.

where $\Omega$ is an arbitrary normalisation constant and $A=E_{g j} / k$. Please note that this approximation is different for each single line of a given ion and that the temperature dependence of $X_{i}(T)$, $\bar{g}(T)$ and $g_{\mathrm{B}}(T)$ is ignored here.

In Fig. 1 the dependence of a given line and thermal bremsstrahlung emission as a function of temperature is given. In our model setup we enrich a fixed space with a fixed mass of metal. Then we construct X-ray emission weighted metallicities, see Eq. (3). This is done by integrating the metallicities along the line of sight and the weighting by the square of the density and the derived temperature dependence, i.e.

Metallicity $_{\mathrm{X} \text {-ray weighted }}=\frac{\sum_{\mathrm{los}} n_{\mathrm{los}}^{2} M_{\mathrm{los}} R(T)_{\mathrm{los}}}{\sum_{\mathrm{los}} n_{\mathrm{los}}^{2} R(T)_{\mathrm{los}}}$,

where $n_{\mathrm{los}}, M_{\mathrm{los}}$ and $R(T)_{\mathrm{los}}$ are the density, metallicity and temperature-dependent weighting factor in each cell along the line of sight. By summing also perpendicular to the line of sight, we can obtain the mean metallicity over the whole cluster.

\section{Spectroscopic metal maps and the comparison with X-ray weighted metal maps}

In order to simulate the X-ray emissivity of our model cluster we use SPEX 2.0 to construct thermal bremsstrahlung spectra including line emission. In Fig. 3a spectrum for the whole model 


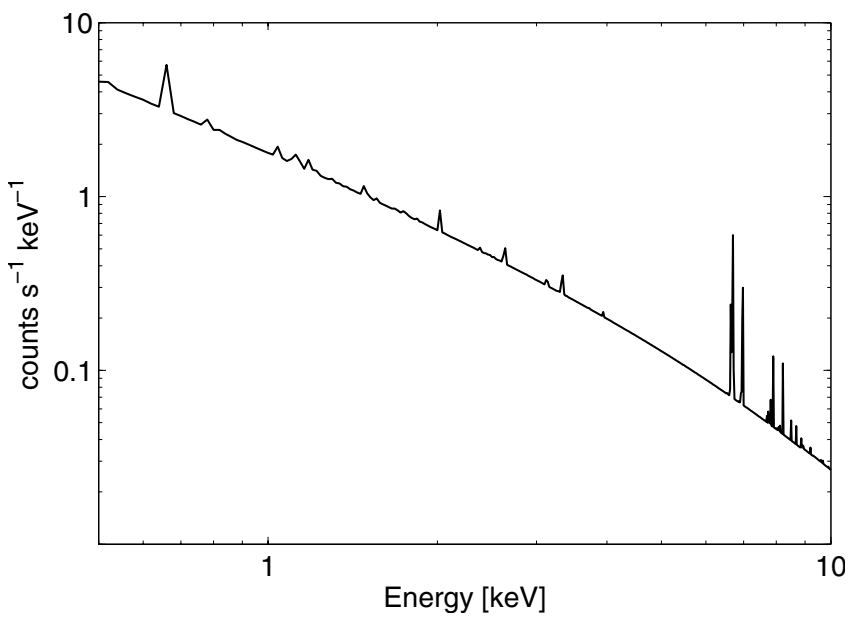

Fig. 3. Model spectrum of our model cluster, obtained with SPEX2.0. The count rate of the whole cluster is shown.
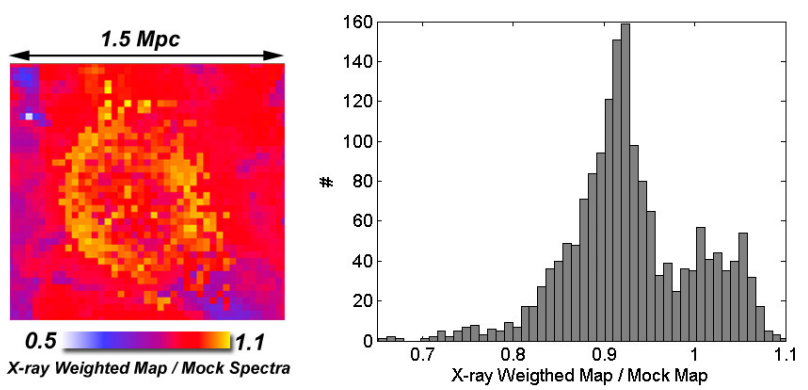

Fig. 4. Ratio of X-ray weighted metal map to synthetic spectroscopic metal map. The image has $1.5 \mathrm{Mpc} h^{-1}$ on the side. Histogram of the left image. The X-ray weighted metal map differs on average by $7 \%$ from the spectroscopic metal map.

cluster is given. For each cell of our simulation, which has a resolution of $128^{3}$, we calculate a model spectrum which we than integrate along the line of sight. By using XSPEC we fit a spectrum to each pixel and hence construct a metallicity map, as shown in Fig. 2.

The model we used in XSPEC12.0 is exactly the same which we used for constructing the synthetic spectra. In order to investigate the quality of our X-ray weighted metal maps we divided the two metal maps, the metal map obtained with XSPEC12.0 by fitting for each pixel the synthetic spectra with a MEKALmodel (thermal bremsstrahlung with line radiation for an optically thin plasma) (Mewe et al. 1985) and the X-ray weighted metal map. The result is shown in Fig. 4, beside the division image a histogram of the ratios is given. The difference of the two maps is on average $7 \%$, leading to the conclusion, that $\mathrm{X}$ ray weighted metal maps are a good approximation for spectroscopic metallicity maps, despite all the approximation made. In the outskirts where the density and temperature of the plasma is lower, the deviation is larger, due to lower metallicities in the Xray weighted metal. This can be understood in terms of the validity of the thermal bremsstrahlung approximation (Eq. (1)), which is only valid for a plasma with temperatures above $3 \times 10^{7} \mathrm{~K}$. In the outskirts of our model cluster $r>600 \mathrm{kpc}$ the temperature can drop below $3 \times 10^{7} \mathrm{~K}$, resulting in a too low metallicity. In this case the synthetic spectra give better results, because the Mekal model is valid for plasma temperatures above $3 \times 10^{4} \mathrm{~K}$ (Mewe et al. 1985). In regions $1 \mathrm{Mpc}$ around massive cluster



Fig. 5. The geometry of the observation and the metal blob positions. The metal blob is placed at different positions along the plotted line, which is perpendicular to the line of sight.

centres the temperature does typically not drop below $3 \times 10^{7} \mathrm{~K}$, therefore the X-ray weighted metal maps used so far to obtain metal maps from simulated clusters are a good approximation.

\section{Results}

\subsection{The mean metallicity as a function of the metal distribution}

As metallicities in the ICM are always obtained by averaging over areas or annuli in the field of view, the question arises, how the real distribution of metals influences the mean metallicity of an observed cluster. Simulations are the only way to address this question, because the 3D metal distribution is exactly known. In order to investigate how sensitive the mean metallicity of the ICM acts on the distribution of heavy elements we investigate an extremely inhomogeneous metal distribution in the ICM. We add metals in a region with $\sim 120 \mathrm{kpc}$ on a side containing $1.17 \times 10^{8} M_{\odot}$ heavy elements, which we place at different positions along a line perpendicular to the line of sight, which passes through the cluster centre, i.e. we always add the same amount of metals to the ICM and see how the mean metallicity of the galaxy cluster in an $1 \mathrm{Mpc}$ radius area depends on the position of the metals. Note that the ICM density distribution corresponds to model cluster A, with zero metallicity, except for the metal box mentioned above. In Fig. 6 the projected mean metallicity of the $\mathrm{ICM}$ in an area with $1 \mathrm{Mpc}$ radius around the cluster centre is shown as a function of the position of the cube with respect to the cluster centre. The ratio between the metal mass obtained from the mean metallicity and the true metal mass is presented as well. The geometry of the observation and the metal cube position is shown in Fig. 5. The metallicity measurements always refer to observations along the line of sight.

It is evident that (see Fig. 6) the position of the cube influences the result dramatically. This can be understood by the density distribution within the ICM. The line flux of a given element can be written as

$F_{\text {line }} \sim \int f(T) n_{\mathrm{e}} n_{\mathrm{i}} \mathrm{d} l \mathrm{~d} A \sim \int f(T) n_{\mathrm{e}} \mathrm{d} M_{\mathrm{i}}$,

where $n_{\mathrm{e}}, n_{\mathrm{i}}$ are the number densities of electrons and the given element ions, $f(T)$ is a temperature dependent emissivity and $\mathrm{d} M_{\mathrm{i}}$ is the differential mass of the given element. In the centre the high density of the ICM results in the highest mean metallicity, which contributes most to the X-ray emission. When the metal blob is placed at a radius of $1 \mathrm{Mpc}$ the mean metallicity drops nearly one order of magnitude, because the outskirts do 




Fig. 6. Overall metallicity of the ICM in a circle with $1 \mathrm{Mpc}$ radius around the cluster centre as a function of the position of a region containing heavy elements. A metal blob with $117 \mathrm{kpc}$ on a side containing $1.17 \times 10^{8} M_{\odot}$ heavy elements moves through the cluster centre, as highlighted in Fig. 5. On the right side the ratio of the metal mass obtained from the mean metallicity and the true 3D metal mass is given. The true metal mass is always higher then the metal mass obtained from X-ray observations. Note that the cluster has zero metallicity, expect for the metal box.

contribute less to the whole cluster emission. From this case of a metal blob placed in primordial ICM we can conclude, that the mean distribution depends strongly on the position of the metal blob within the ICM.

Recalculating the mass of metals residing in the ICM from the mean metallicity, shows the same dependence, see Fig. 6. The mean metallicity always underestimated the metal mass present in the model cluster. The discrepancy is a factor of 10 in the centre and increases to a factor of 100 in the outskirts. The reason therefore is again the fact that emissivity by thermal bremsstrahlung is mainly driven by the density of the ICM, which decreases from the cluster centre to the outskirts by several magnitudes and the inhomogeneous metal distribution. The integration of all quantities along the line of sight and the investigated area leads to the discrepancy between the true 3D metal mass and the metal mass obtained by the mean metallicity of the ICM. This is of course an extreme example, but it demonstrates how strong the mean metallicity of a whole cluster depends on the actual metal distribution. In the case of a constant metallicity over the whole ICM, the metal mass obtained by the mean metallicity in a circle of $1 \mathrm{Mpc}$ radius around the cluster centre is of course exactly the same as the true $3 \mathrm{D}$ metal mass.

\subsection{The mean metallicity as a measure for the true metal mass}

In order to test, whether the mean metallicity in a defined region around the cluster centre represents a good measure for the true metal mass within this area or not, we performed several tests with the model clusters. The metal content in the model galaxy clusters were calculated from an enrichment process investigation, where galactic winds and ram-pressure stripping acts simultaneously to enrich the ICM. The simulation starts at a redshift of $\sim 20$ and results in a metal distribution within the ICM as shown in Fig. 7. For more details on the enrichment processes and the metal distribution see Kapferer et al. (2007). At redshift $z=0$ we extract the mean metallicity within circles of different



Fig. 7. Metallicity profiles for model clusters A and B.

radii around the cluster centre $(100,200,500$ and $800 \mathrm{kpc})$ and extract from the 3D simulation the mean ICM density within the corresponding spheres. As we know exactly the amount and distribution of heavy elements, we can compare these two quantities, i.e. the true heavy element mass and the X-ray measured heavy element mass. In Fig. 8 the spatial distribution of the density, temperature and metallicity of model cluster A is presented. Whereas the density does not show significant substructures, the metallicity is inhomogeneous.

In Table 1 the mean metallicity, true metal and X-ray measured metal mass for different circles around the cluster centre for model cluster A and B are given. We find, that the X-ray measured metal mass always results in lower heavy element masses compared to the true metal mass in the same region of interest. If the radius is smaller, the discrepancy can be up to three times more metals in the ICM, than measured by X-ray spectra. By increasing the radius, i.e. averaging over much larger volumes, the discrepancy becomes smaller. At a radius at $800 \mathrm{kpc}$ the X-ray measured heavy element mass gives $20 \%$ to $50 \%$ lower masses than the true metal mass. In Fig. 9 two metal-mass profiles for model cluster A are shown. The metal mass increases in general with increasing radius, because the volume of the radial shell increases with the square of the distance to the cluster centre. Although the metallicity decreases at larger radii, the larger volume results in a higher metal mass. The metal mass X-ray profile (dashed line) is considerably lower then the true metal mass profile. The discrepancy arises by averaging of the metallicity in annuli at all radii, which wipes out very prominent metallicity peaks all over the cluster. In Fig. 8 the distribution of the density, temperature and metallicity of the ICM in model cluster A at redshift 0 is shown. The high metallicity regions in the outskirts are clearly visible in the 3D metal distribution. The inhomogeneous metal distribution in galaxy clusters leads to a systematic underestimate of the metal mass by X-ray observations. For example the ratio of the integrated true metal-mass profile to the integrated X-ray metallicity obtained metal-mass profile in an $200 \mathrm{kpc}$ radius is 2.70 . Investigations of the amount of metals residing in galaxies in galaxy clusters and in the ICM (e.g. Renzini et al. 1993) do always underestimate the true metal mass within the ICM. These works concluded, that on average the amount of heavy elements within galaxies and the ICM is comparable, would get a shift towards the conclusion, that even more metal mass resides within the ICM as in the galaxies. 


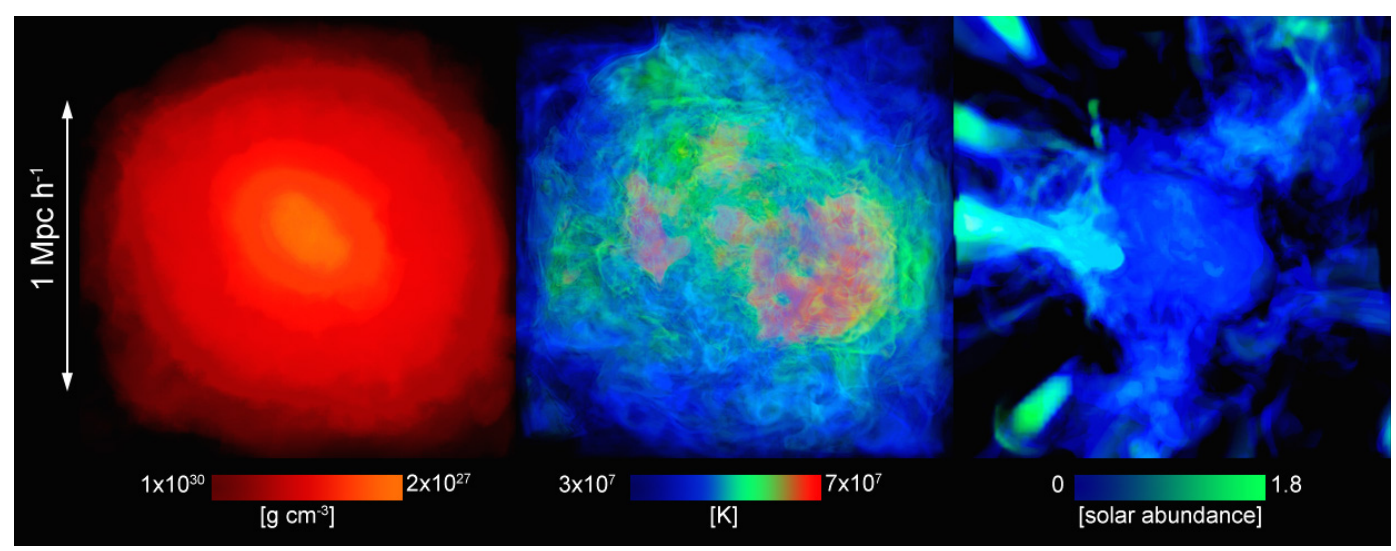

Fig. 8. Three dimensional distribution of the density, temperature and the metallicity of the ICM of model cluster A.

Table 1. True metal mass versus X-ray obtained metal mass in different spheres around the cluster centre for a less massive model galaxy cluster A and a massive model galaxy cluster $\mathrm{B}$.

\begin{tabular}{cccccc}
\hline \hline & & & & Mean metallicity & Ratio \\
Model cluster & Radius $[\mathrm{kpc}]$ & True metal mass $\left[M_{\odot}\right]$ & X-ray measured metal mass $\left[M_{\odot}\right]$ & Solar abundance & C.3 to C.4 \\
\hline A & 800 & $1.07 \times 10^{10}$ & $8.40 \times 10^{9}$ & 0.346 & 1.27 \\
A & 500 & $4.50 \times 10^{9}$ & $2.63 \times 10^{9}$ & 0.356 & 1.71 \\
A & 200 & $6.05 \times 10^{8}$ & $2.24 \times 10^{8}$ & 0.370 & 2.70 \\
A & 100 & $9.70 \times 10^{7}$ & $3.10 \times 10^{7}$ & 0.383 & 3.13 \\
\hline B & 800 & $1.02 \times 10^{11}$ & $6.70 \times 10^{10}$ & 0.94 & 1.52 \\
B & 500 & $4.70 \times 10^{10}$ & $2.75 \times 10^{10}$ & 0.95 & 2.80 \\
B & 200 & $7.50 \times 10^{9}$ & $3.45 \times 10^{9}$ & 0.96 & 2.17 \\
B & 100 & $1.25 \times 10^{9}$ & $5.40 \times 10^{8}$ & 0.97 & 2.31 \\
\hline
\end{tabular}



Fig. 9. Metal mass profile per radial bin for model cluster A. Solid line: true metal mass within a three dimensional radial bin. Dashed line: metal mass obtained by multiplying the metallicity profile taken from the X-ray weighted metal map (Eq. (4)) with the three dimensional gas mass within the same bin (bin size $80 \mathrm{kpc}$ ).

\section{Discussion and conclusions}

We investigate the quality of the metallicities obtained by X-ray observation of the ICM as a measure for the true metal mass of the ICM. In addition we test if X-ray weighted metal maps of galaxy cluster simulations are comparable to synthetic X-ray spectra composed of thermal bremsstrahlung and line radiation. The results of our work are as follows:

- If the ICM temperature is above $T>3 \times 10^{7} \mathrm{~K}$ X-ray weighted metal maps results nearly in the same metallicities as obtained from synthetic X-ray spectra. The difference is on average less than $7 \%$ in areas with $r<750 \mathrm{kpc}$ around the centre of galaxy clusters.

- We test how much the distribution of metals within a sphere of $r=1 \mathrm{Mpc}$ around the cluster centre changes the X-ray measured mean metallicity of this area. We found that the X-ray obtained mean metallicity can change by a factor of 10 in the extreme case of a distinct metal blob residing in primordial ICM, depending on the distance from the cluster centre.

- By using realistic metal distributions in simulated galaxy clusters we test the quality of the X-ray obtained overall metallicity as a measure for the metal mass. We found that depending on the size of the investigated area around the cluster centre the true metal mass can be three times higher than the metal mass obtained by X-ray observations. If the radius increases the discrepancy gets smaller, but in all cases the true metal mass is higher than the metal mass obtained by X-ray observations.

The discrepancies of the true metal mass and the X-ray obtained metal mass within the ICM in galaxy clusters can be understood in terms of averaging. The observer measures integrated quantities along the line of sight, including dense regions in the centre and less dense regions in the outskirts. In order to be able to get reasonable X-ray spectra of the ICM, observers must always collect X-ray photons over an area. As the metallicity is not constant throughout the galaxy cluster, i.e. the ratio of primordial 
ICM to heavy elements is not constant, the integration of thermal bremsstrahlung and line radiation results in different ratios, leading to too low metal masses. Applying multi-temperature instead of single-temperature models for the ICM would alleviate the problem partially as shown by Buote \& Canizares (1994).

Taking the results of this work into account, it is obvious that estimates from X-ray observations on the heavy elements (e.g. iron) content in the ICM lead to masses, which are too low.

Acknowledgements. The authors thank the anonymous referee for fruitful comments, which helped to improve the paper. The authors would like to thank Etienne Pointecouteau for the useful help with XSPEC12.0 and Jelle Kaastra for the help regarding SPEX2.0. The authors acknowledge the Austrian Science Foundation (FWF) through grants P18523-N16 and P19300-N16. Thomas Kronberger is a recipient of a DOC-fellowship of the Austrian Academy of Sciences. In addition, the authors acknowledge the ESO Mobilitätsstipendien des BMWF (Austria), the Tiroler Wissenschaftsfonds (Gefördert aus Mitteln des vom Land Tirol eingerichteten Wissenschaftsfonds) and the UniInfrastrukturprogramm 2005/06 from the BMWF.

\section{References}

Anders, E., \& Grevesse, N. 1989, Geochim. Cosmochim. Acta, 53, 197 Baumgartner W. H., Loewenstein M., Horner D. J., \& Mushotzky R. F. 2005, ApJ, 620, 680

Buote, D. A., \& Canizares, C. R. 1994, ApJ, 427, 86
Cora, S. A. 2006, MNRAS, 368, 1540

Domainko, W., Mair, M., Kapferer, W., et al. 2006, A\&A, 452, 795

Dorman, B., Arnaud, K. A., \& Gordon, C. A. 2003, BAAS, 35, 641

Durret, F., Lima Neto, G. B., \& Forman, W. 2005, A\&A, 432, 809

Ettori S., Fabian A. C., Allen S. W., \& Johnstone R. 2002, MNRAS, 331, 635

Finoguenov A., Matsushita K., Böhringer H., Ikebe Y., \& Arnaud M. 2002, A\&A, 381, 21

Kaastra, J. S., Mewe, R., \& Nieuwenhuijzen, H. 1996, UV and X-ray Spectroscopy of Astrophysical and Laboratory Plasmas: Proceedings of the Eleventh Colloquium on UV and X-ray ... held on May 29-June 2, 1995, Nagoya, Japan, ed. K. Yamashita \& T. Watanabe (Tokyo: Universal Academy Press), Frontiers Sci. Ser., 15, 411

Kapferer, W., Ferrari, C., Domainko, W., et al. 2006, A\&A, 447, 827

Kapferer, W., Kronberger, T., Weratschnig, J., et al. 2007, A\&A, 466, 813

Mewe, R., Gronenschild, E. H. B. M., \& van den Oord, G. H. J. 1985, A\&AS, 62, 197

Mitchell, R. J., Culhane, J. L., Davison, P. J. N., \& Ives, J. C. 1976, MNRAS, $175,29 \mathrm{P}$

Moll, R., Schindler, S., Domainko, W., et al. 2007, A\&A, 463, 513

Mushotzky, R. 1999, The High-redshift Universe: Galaxy Formation and Evolution at High Redshift, Proceedings of a conference held in Berkeley, CA, 21-24 June, 1999, ed. A. J. Bunker \& W. J. M. van Breugel, ASP Conf. Proc., 193, 651

Pratt, G. W., Boehringer, H., Croston, J. H., et al. 2006, A\&A, 461, 71

Renzini, A., Ciotti, L., D’Ercole, A., \& Pellegrini, S. 1993, ApJ, 419, 52

Sanders, J. S., Fabian A. C., Allen S. W., \& Schmidt, R. W. 2004, MNRAS, 349, 952

Schindler, S., Kapferer, W., Domainko, W., et al. 2005, A\&A, 435, L25

Springel, V., \& Hernquist, L. 2003, MNRAS, 339, 289 